\title{
Spontaneous spatial fractal pattern formation in absorptive systems
}

\author{
J. G. Huang, \\ Faculty of Advanced Technology, University of Glamorgan, \\ Pontypridd CF37 1DL, United Kingdom \\ J. M. Christian and G. S. McDonald \\ Joule Physics Laboratory, School of Computing, Science and Engineering, \\ Materials \& Physics Research Centre, University of Salford, \\ Greater Manchester M5 4WT, United Kingdom
}

Submitted to Journal of Nonlinear Optical Physics and Materials as a Rapid Communication

$13^{\text {th }}$ March 2012

\begin{abstract}
We predict, for the first time to our knowledge, that purely-absorptive nonlinearity can support spontaneous spatial fractal pattern formation. A passive optical ring cavity with a thin slice of saturable absorber is analyzed. Linear stability analysis yields threshold curves for Turing (static) instabilities with features proposed as characteristics of potential fractal pattern formation. Numerical simulations of the fully-nonlinear dynamics, with both one and two transverse dimensions, confirm theoretical predictions.
\end{abstract}

PACS numbers: 42.65.-k, 42.65.Jx, 42.65.Ky, 42.65.Pc, 42.65.Sf.

Keywords: $\quad$ spontaneous patterns, Turing instability, spatial fractals, saturable absorber, ring cavities 
Spontaneous pattern formation appears in a wide range of disciplines, including physics, chemistry, biology, and engineering [1,2]. The universal phenomena of symmetry-breaking and self-organization often conspire to drive the emergence of one of two generic spatial structures: (i) simple Turing-instability patterns with a single dominant scalelength (e.g., hexagons, squares, stripes, and rings); (ii) complex patterns with proportional levels of detail spanning decades of scale (fractals, i.e., inherently scaleless objects).

Previously, a connection was sought between these two apparently exclusive signatures of complexity [3]. A characteristic was proposed as capable of predicting the fractal-generating capacity of wave-based nonlinear systems: systems whose static Turing instability threshold spectrum exhibit a large number of comparable frequency minima are potentially capable of generating spontaneous fractal structures. This type of pattern formation was confirmed in a simple dispersive system: the classic diffusive-Kerr slice with a single feedback mirror [2,4]. In this Communication, we predict the first spontaneous spatial fractals in a purelyabsorptive nonlinear ring cavity. Such a choice of system enables us to test, simultaneously, the independence of the proposed fractal-generating mechanism with respect to both the nature of the nonlinearity and the particulars of the experimental configuration (e.g., single feedback-mirror, ring cavity, etc.).

The linear fractals found in the transverse eigenmodes of some unstable cavity lasers are formed through the interplay between diffraction and successive round-trip magnifications [5-7]. The multiscale origin of those patterns lies in a linear superposition of images, each of which has a larger scalelength than the preceding one. Here, the cascade to fractality is driven solely by intrinsic nonlinear dynamics. Above the Turinginstability thresholds, nonlinear cascades contribute to the excitation of higher spatial frequencies, leading to smaller-scale details in the pattern. Such nonlinear fractals are truly spontaneous spatial structures (emerging in homogeneous systems) and physically distinct from "soliton fractals", where each new scalelength is introduced by an individual, abrupt material inhomogeneity [8].

Over recent decades, nonlinear ring cavities have provided a paradigm for studying optical pattern formation. Many analyses have simplified the full spatiotemporal dynamics by adopting the mean-field limit. However, Turing-instability threshold spectra obtained from mean-field models typically possess only a single minimum, and thereby preclude predictions of cascades-to-fractality. Allowing for light propagation effects reveals possibilities of multiple emergent spatial frequencies $[9,10]$. The generality of the proposed fractalgenerating signature [3] is tested here by examining a configuration (see Fig. 1) that is quite different from that in 
Ref. [3]. We consider a thin slice of saturable absorber with a near-negligible width $l$ (thus avoiding detailed nonparaxial treatment of small-scale field structure). We further assume that the medium polarization relaxes sufficiently rapidly (compared to the dynamics of the population inversion $w$ and the cavity transit time $t_{R}$ ) that it can be adiabatically eliminated. When potential diffusion of $w$ is accounted for, the longitudinal ( $z$ ) evolution of the electric field envelope $E$ and medium dynamics are governed by

$$
\begin{gathered}
\frac{\partial E}{\partial z}=\left(\frac{\alpha_{0}}{2}\right) \frac{E w}{1+i \Delta}, \\
T_{1} \frac{\partial w}{\partial t}-l_{D}^{2} \nabla_{\perp}^{2} w+(1+w)=-\frac{T_{1} T_{2}}{1+\Delta^{2}}|E|^{2} w
\end{gathered}
$$

where $\nabla_{\perp}^{2}$ is a transverse Laplacian, $T_{1}$ and $T_{2} \ll T_{1}$ are relaxation times for $w$ and the polarization, respectively, $l_{D}$ is a diffusion length, $\alpha_{0}$ an absorption coefficient, and $\Delta$ the detuning between pump and atomic resonance frequencies. The system can be either purely absorptive $(\Delta=0)$ or purely dispersive $(|\Delta| \gg 1)$.

Denoting the Fourier transform of $E$ at the output face of the slice by $\tilde{E}(\mathbf{K}, l, t)$, model (1) is supplemented by a ring-cavity boundary condition [9]

$$
\tilde{E}(\mathbf{K}, 0, t)=a \delta(\mathbf{K})+R^{1 / 2} \exp \left(i \phi_{0}\right) F\left(K, K_{C}\right) \exp \left[-i \theta\left(K^{2}\right)\right] \tilde{E}\left(\mathbf{K}, l, t-t_{R}\right),
$$

where $\mathbf{K}$ is transverse wavevector, $a$ is intracavity plane-wave pump amplitude, $\delta(\mathbf{K})$ is a Dirac delta function, $R$ is the intensity reflectivity of the coupling mirror [2] (other mirrors are assumed lossless) and $\phi_{0}$ is cavity mistuning. The phase factor $\theta\left(K^{2}\right)=2 \sigma K^{2} /\left[1+\left(1-K^{2} / k_{0}^{2}\right)^{1 / 2}\right]$ allows for Helmholtz diffraction [11] in the freespace path, where $\sigma \equiv L / 2 k_{0}$, and $k_{0}$ is the carrier wavenumber. The function $F\left(K, K_{C}\right)$ represents a spatial filter whose effect, in combination with diffraction, provides a continuously-variable cut-off frequency $K_{C}$ :

$$
F\left(K, K_{C}\right) \exp (-i \theta)=\exp \left[-i \sigma \frac{2 K^{2}}{1+\sqrt{1-\left(K / K_{C}\right)^{2}}}\right]
$$

When letting $k_{0} \rightarrow \infty$, the paraxial propagation factor $\theta\left(K^{2}\right) \simeq \sigma K^{2}$ is recovered so that, when $F\left(K, K_{C}\right)=1$, Eq. (2) becomes the classic (paraxial) boundary condition [9]. 
Linear stability analysis has been performed on the steady-state, transversely-homogeneous solutions $\left(E_{0}, w_{0}\right)$ of system (1), subject to the paraxial boundary condition [9]. Perturbations are assumed proportional to $\exp (i \mathbf{K} \cdot \mathbf{x}+\lambda t)$, where $\lambda \equiv \Lambda-i \Omega, \Lambda$ is the growth rate, and $\Omega$ the Hopf frequency. The threshold for spontaneous static patterns is found when $\lambda=0$, yielding a condition on the intracavity intensity $I_{t h} \equiv\left|E_{0}\right|^{2}$ :

$$
\frac{\delta+T_{1} T_{2} I_{t h}}{\delta}+l_{D}^{2} K^{2}=\frac{T_{1} T_{2} I_{t h} \alpha_{0} l \sqrt{r}}{\delta\left(1+T_{1} T_{2} I_{t h}\right)} \frac{\cos \Gamma_{t h}+\Delta \sin \Gamma_{t h}-\sqrt{r}}{1+r-2 \sqrt{r} \cos \Gamma_{t h}},
$$

where $\delta \equiv 1+\Delta^{2}, \Gamma_{t h} \equiv \phi_{0}+\alpha_{0} l \Delta\left(\delta+T_{1} T_{2} I_{t h}\right)-\sigma K^{2}$ and $r \equiv R \exp \left[-\alpha_{0} l /\left(\delta+T_{1} T_{2} I_{t h}\right)\right]$. Threshold condition (4) divides the $\left(I_{t h}, K\right)$ plane into a large number of islands (see Fig. 2). With increasing $K$, the width and separation of the islands decrease while the minimum (maximum) thresholds increase (decrease) smoothly. Sufficiently small $l_{D}$ allows the coexistence of large number of comparable instability islands. This multiscale characteristic may indicate spontaneous fractal patterns [3].

In the absence of diffusion, system (1) has a global instability minimum [see Fig. 2(b)]. When the pump intensity just exceeds threshold, spatial frequencies defined by this minimum all have the same growth rate. One then expects that the resultant pattern in the one-dimensional transverse plane will be an extremely complicated area-filling pattern with fractal dimension 2 . To test this conjecture, the stationary state of system (1) is initialized above threshold, and with a $0.1 \%$ level of background noise (added to accelerate the pattern formation process). For simple pattern formation, the filter $K_{C}$ is set so that only those waves within the first instability island may propagate freely around the cavity (spectral components with $K>K_{C}$ are attenuated). The static Turing intensity pattern $I(x) \equiv|E(x)|^{2}$ that eventually emerges has a single well-defined scale-length, and its corresponding power spectrum $P(K)$ contains a dominant peak plus a set of weaker harmonics [see Fig. 3(a)]. When the filter is removed (by setting $K_{C}=k_{0}=11,200$ ), waves associated with many more instability minima may propagate and interact. Intrinsic nonlinear dynamics (e.g., harmonic generation and four-wave mixing cascades) then lead to rapid growth at the high- $K$ end of the power spectrum [this process is illustrated in Figs. 3(b) and 3(c)]. After a sufficient number of transits, all spatial frequencies are present in the pattern $I(x)$ with roughly the same strength, resulting in an area-filling fractal (with dimension 2) that possess comparable levels of structure down to spatial scales at the optical wavelength, i.e. $\sim 2 \pi / k_{0}$ [see Fig. 3(d)]. 
The roughness-length dimension $D_{r l}$ of the fractal pattern in Fig. 3(d) can be calculated [12] from $D_{r l}=$ $2-d \ln <S(W)>d d(\ln W)$. For an interval $W$ of a curve $I(x)$, the root-mean-square (RMS) roughness $S(W)$ is the standard deviation of the first differences $\Delta I$ of the values of $I$ within the interval, $\Delta I(x) \equiv I(x)-I(x-\Delta x)$, and $\Delta x$ is the constant sampling interval of the dataset representing the curve. Here, $\langle S(W)>$ denotes the average value of $S(W)$ over a number of intervals. Figure 4 shows the RMS roughness for the curve in Fig. 3(d); the main portion of the plot has a slope of zero, and thus $D_{r l}=2$. This result is in full agreement with predictions from the linear stability analysis. Although $D_{r l}$ is an integer, the pattern itself is still a fractal because its fractal dimension (i.e., $\left.D_{r l}=2\right)$ is larger than its topological dimension [i.e., 1 for the pattern in Fig. 3(d)].

One may now consider spontaneous pattern formation when two transverse directions are present. The evolution of the perturbed stationary state toward a simple (static) pattern - in this case, a hexagonal array - is shown in Fig. 5. The hexagon patterns are reminiscent of the classic patterns observed by Grynberg et al. [13]. Once this static pattern has been reached, the filter is removed by setting $K_{C}=k_{0}=90$ and subsequent evolution is monitored. Intermediate patterns form that resemble the superlattice structures observed in optical feedback experiments with Kerr-like nonlinearities [14,15]. Our simulated patterns then subsequently develop an increasing level of fine structure (see Fig. 6). However, accurate 2D simulations of fractal (as opposed to simple) patterns are exceptionally resource-hungry: computer memory limitations restrict $k_{0}$ to relatively small values compared to $1 \mathrm{D}$ computations [16] and prevent the final pattern reaching a truly volume-filling character (that should have dimension 3).

In conclusion, it has been shown that a purely-absorptive nonlinear system can give rise to spontaneous spatial fractal patterns. Linear analysis reveals Turing-instability threshold spectra with characteristics suggesting cascades-to-fractality. Simulations demonstrate the generality of this proposed fractal-generating signature [3], i.e., independence with respect to both system nonlinearity and the details of experimental geometry (e.g., single feedback-mirror or ring cavity). We expect other photonic systems with similar Turing threshold spectra to be capable of generating spontaneous fractal patterns. Any experiment designed to observe spontaneous spatial optical fractals will inevitably involve some high-frequency cut-off (e.g., intrinsic filtering due to finite beamsize considerations, while simulations presented here and elsewhere [3] have considered plane-wave pumping only), and such effects may prevent patterns in the laboratory from reaching their area-filling or volume-filling potential. However, the results reported in this Communication (extending considerations from single-feedback- 
Page 6 of 15

mirror Kerr configurations to absorptive ring-cavity geometries) support the notion of universality of the proposed mechanism for predicting a system's fractal-generating capacity. 


\section{REFERENCES}

[1] M. C. Cross and P. C. Hohenberg, Pattern formation outside of equilibrium, Rev. Mod. Phys. 65 (1993) 851.

[2] F. T. Arecchi, S. Boccaletti, and P. Ramazza, Pattern formation and competition in nonlinear optics, Phys. Rep. 318 (1999) 1.

[3] J. G. Huang and G. S. McDonald, Spontaneous optical fractal pattern formation, Phys. Rev. Lett. 94 (2005) 174101.

[4] G. D'Alessandro and W. J. Firth, Spontaneous hexagon formation in a nonlinear optical medium with a feedback mirror, Phys. Rev. Lett. 66 (1991) 2597.

[5] G. P. Karman and J. P. Woerdman, Fractal structure of eigenmodes of unstable-cavity lasers, Opt. Lett. 23 (1998) 1909.

[6] G. P. Karman, G. S. McDonald, G. H. C. New, and J. P. Woerdman, Fractal modes in unstable resonators, Nature 402 (1999) 138.

[7] M. Berry, C. Storm, and W. V. Saarloos, Theory of unstable laser modes: edge waves and fractality, Opt. Commun. 197 (2001) 393.

[8] S. Sears, M. Soljacic, M. Segev, D. Krylov, and K. Bergman, Cantor set fractals from solitons, Phys. Rev. Lett. 84 (2000) 1902.

[9] D. W. McLaughlin, J. V. Moloney, and A. C. Newell, New class of instabilities in passive optical cavities, Phys. Rev. Lett. 54 (1985) 681.

[10] A. S. Patrascu, C. Nath, M. Le Berre, A. Ressayre, and A. Tallet, Multi-conical instability in the passive ring cavity: linear analysis, Opt. Commun. 91 (1992) 433.

[11] M. D. Feit and J. A. Fleck, Beam nonparaxiality, filament formation, and beam breakup in the selffocusing of optical beams, J. Opt. Soc. Am. B 5 (1988) 633.

[12] BENOIT 1.3, TruSoft International Inc. http://www.trusoft.netmegs.com/benoit.html. 
[13] G. Grynberg, E. LeBihan, P. Verkerk, P. Simoneau, J. J. R. Leite, D. Bloch, S. Le Boiteaux, and M. Ducloy, Observation of instabilities due to mirrorless four-wave mixing oscillation in sodium, Opt. Commun. 67 (1988) 363.

[14] E. Pampaloni, S. Residori, S. Soria, and F. T. Arecchi, Phase locking in nonlinear optical patterns, Phys. Rev. Lett. 78 (1997) 1042.

[15] S. Residori, Patterns, fronts and structures in a liquid-crystal-light-valve with optical feedback, Phys. Rep. $416(2005) 201$.

[16] To maintain high numerical accuracy, $k_{0}$ must be less than one half of the maximum spatial frequency that can be represented on the computational grid. In this way, one avoids various numerical artefacts, such as aliasing, that would otherwise invalidate our results. 


\section{FIGURE CAPTIONS}

Fig. 1. (Color online) Schematic diagram of the nonlinear ring cavity geometry with a spatial frequency filter $F\left(K, K_{C}\right)$.

Fig.2. (Color online) Multi-Turing threshold instability spectra for the purely-absorptive $(\Delta=0)$ ringcavity system (1) with (a) a finite level of diffusion $\left(l_{D}=0.2\right)$ and (b) no diffusion $\left(l_{D}=0\right)$. Other parameters are set to: $a=19.0, \phi_{0}=\pi / 4, R=0.9, \sigma=1.0, \alpha_{0} l=10.0, T_{1}=1.0$ and $T_{2}=T_{1} / 100$.

Fig. 3. (Color online) Evolution of one-dimensional pattern (first column) and its corresponding power spectrum (second column) in a purely absorptive cavity containing a thin slice of material. Other parameters are the same as in Fig. 2(a). (a) Static pattern (formed when the filter is set to $K_{C}=1.0$ ) when the filter is removed at time $t=0$. Subsequent patterns after the filter is removed $\left(K_{C}=k_{0}=\right.$ 11,200) shown for times: (b) $t=500 t_{R}$, (c) $t=1,000 t_{R}$, and (d) $t=3,000 t_{R}$.

Fig. 4. (Color online) Root-mean-square roughness of the real-space pattern $I(x)$ shown in Fig. 3(d).

Fig.5. (Color online) Formation of a simple Turing (hexagon) pattern in purely absorptive cavity with two transverse dimensions, and where the filter has been set to $K_{C}=1.0$. All other parameters are the same as those in Fig. 2(a). Patterns are shown for times: (a) $t=t_{R}$, (b) $t=280 t_{R}$, (c) $t=360 t_{R}$, and (d) $t$ $=900 t_{R}$.

Fig. 6. (Color online) Evolution of the hexagonal array from Fig. 5(d) toward a fractal. (a) Initial simple hexagonal pattern at time-zero (when the filter is removed by setting $K_{C}=k_{0}=90.0$ ). Patterns are shown for times: (b) $t=270 t_{R}$, (c) $t=350 t_{R}$, (d) $t=860 t_{R}$. 


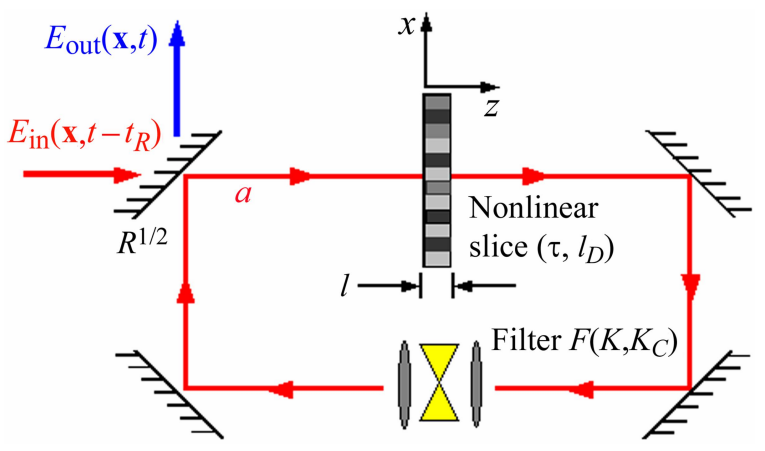

J. G. Huang, J. M. Christian, and G. S. McDonald,

Spontaneous spatial fractal pattern formation in absorptive systems,

Figure 1. 



J. G. Huang, J. M. Christian, and G. S. McDonald,

Spontaneous spatial fractal pattern formation in absorptive systems,

Figure 2. 
(a)

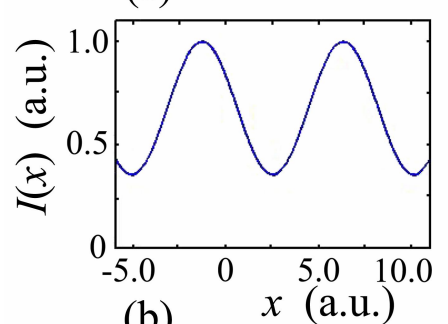

(b)

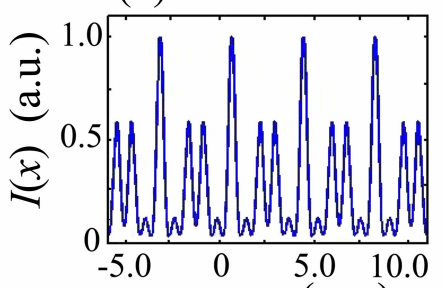

(c) $x$ (a.u.)

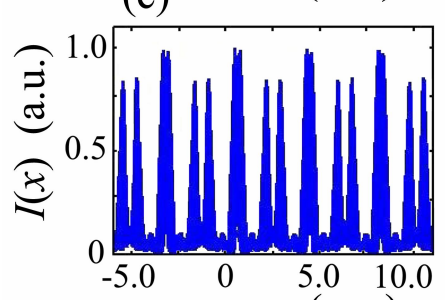

(d) $\quad x$ (a.u.)

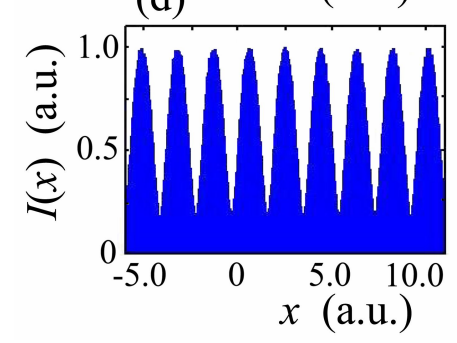

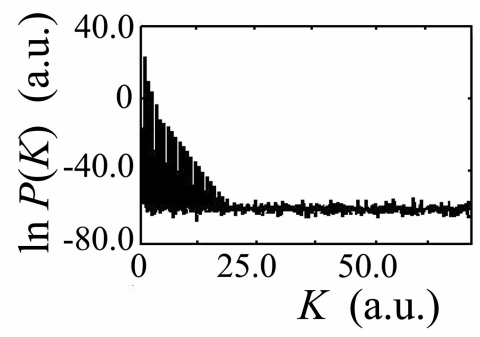
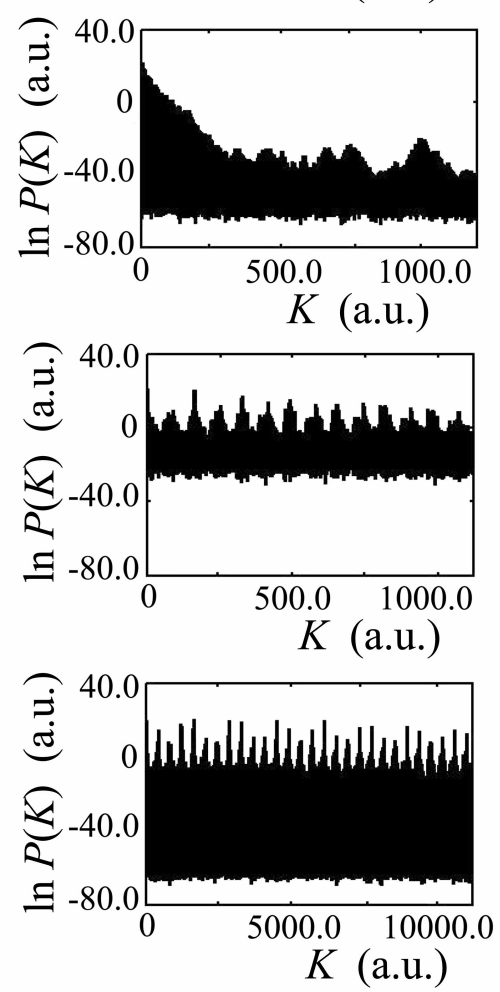

\section{J. G. Huang, J. M. Christian, and G. S. McDonald,}

Spontaneous spatial fractal pattern formation in absorptive systems,

Figure 3. 




J. G. Huang, J. M. Christian, and G. S. McDonald,

Spontaneous spatial fractal pattern formation in absorptive systems,

Figure 4. 


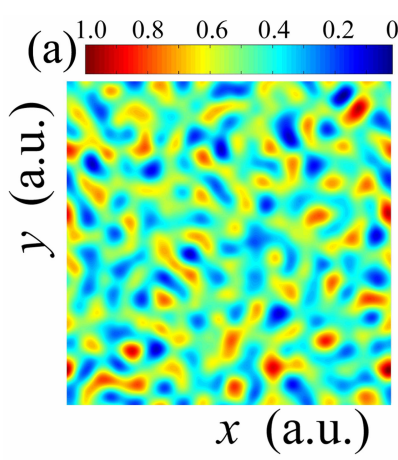

(b) $\begin{array}{llllll}1.0 & 0.8 & 0.6 & 0.4 & 0.2 & 0\end{array}$



(c)

(d) $\begin{array}{llllll}1.0 & 0.8 & 0.6 & 0.4 & 0.2 & 0 \\ & & & & \end{array}$

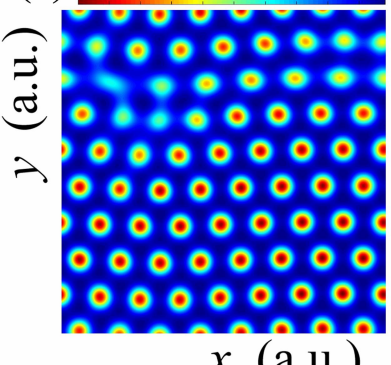

(50 000000

戸 00000000

$\underbrace{\mathbb{E}}_{0} 0000000$

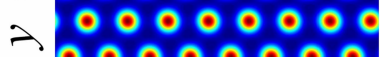

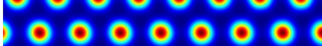

0 ० 0 0 0000

p 0 ० 0 0 000

$x$ (a.u.)

J. G. Huang, J. M. Christian, and G. S. McDonald,

Spontaneous spatial fractal pattern formation in absorptive systems,

Figure 5. 

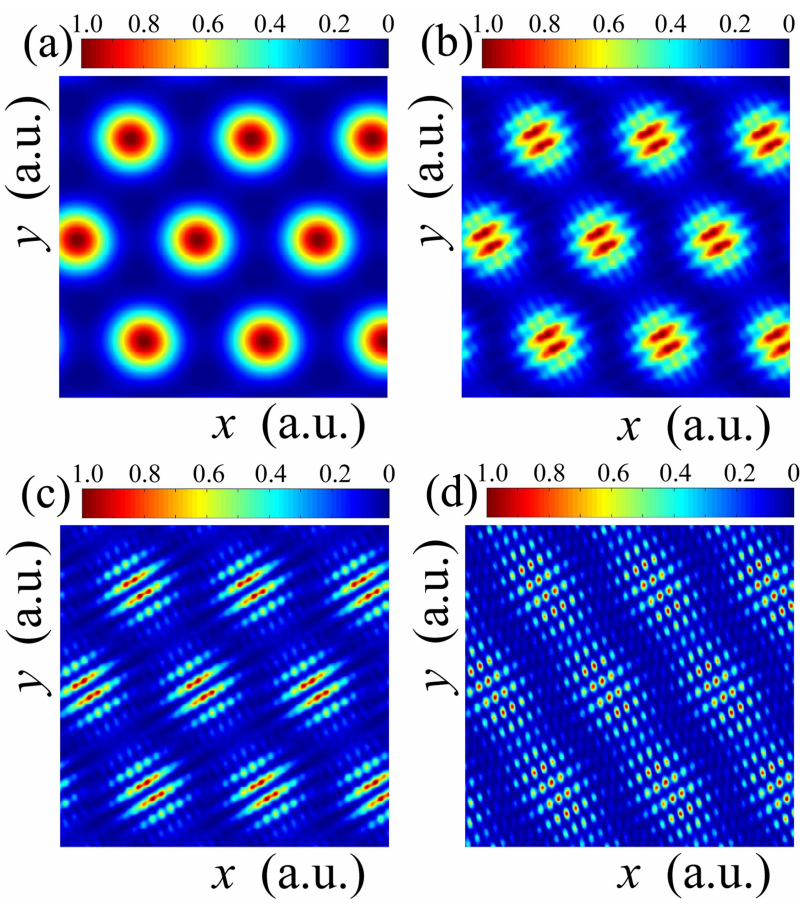

J. G. Huang, J. M. Christian, and G. S. McDonald,

Spontaneous spatial fractal pattern formation in absorptive systems,

Figure 6. 